\title{
Modeling Static Electric Field Effect on Nematic Liquid Crystal Director Orientation in Side-Electrode Cell
}

\author{
Alexander Ayriyan ${ }^{1, \star}$, Edik Ayryan ${ }^{1,2}$, Alexandre Egorov ${ }^{3, \star \star}$, Maria Dencheva-Zarkova ${ }^{4}$, \\ Georgi Hadjichristov ${ }^{4}$, Yordan Marinov ${ }^{4}$, Igor Maslyanitsyn ${ }^{3}$, Alexander Petrov ${ }^{4}$, Lidia Popova ${ }^{4}$, \\ Vladimir Shigorin ${ }^{3}$, Alfredo Strigazzi ${ }^{5,6}$, and Sofia Torgova ${ }^{7}$ \\ ${ }^{1}$ Joint Institute for Nuclear Research, Dubna, Russia \\ ${ }^{2}$ Peoples' Friendship University of Russia (RUDN University), Moscow, Russia \\ ${ }^{3}$ Prokhorov General Physics Institute, Russian Academy of Sciences, Moscow, Russia \\ ${ }^{4}$ Georgi Nadjakov Institute of Solid State Physics, Bulgarian Academy of Sciences, Sofia, Bulgaria \\ ${ }^{5}$ Physics Institute of Condensed Matter and Complex Systems, CNISM, Politecnico di Torino, Torino, Italy \\ ${ }^{6}$ National Research Nuclear University MEPhI (Moscow Engineering Physics Institute), Moscow, Russia \\ ${ }^{7}$ Lebedev Physical Institute, Russian Academy of Sciences, Moscow, Russia
}

\begin{abstract}
A two-dimensional model of Fredericks effect was used for the investigation of the static electric field influence on nematic liquid crystal director orientation in the side-electrode cell. The solutions of the equations describing the model were obtained by finite-difference methods. Fredericks transition threshold for the central part of the cell, as well as dependencies of the distribution of the director orientation patterns on the electric field and location, were obtained. The numerical results are found to agree qualitatively with the experiment. Further investigations are needed to elucidate completely the Fredericks effect.
\end{abstract}

\section{Introduction}

A change of the preferential orientation of liquid crystal (LC) molecules (director), in an electric or magnetic field exceeding a threshold value, results in significant change of LC properties. This effect (known as orientation Fredericks transition) is used in numerous applications and extensively studied [1]. LC cells with plane parallel glass platelets with thin LC layer between them and side electrodes are used in numerous experiments devoted to electro-optical properties of LC (see e.g. [2, $3])$.

Such cells were used for studies of second harmonic generation in samples of nematic liquid crystal (NLC) 4-n-pentyl-4'-cyanobiphenyl (5CB) [3]. Domain formation with quite different director orientations has been found to occur when a high pulse-periodic voltage was applied to the electrodes. This is to some extent inconsistent with the common point of view that changes in the NLC director orientation are smooth. The discovered effect can be used for a number of applications and therefore, its investigation is of great interest.

\footnotetext{
^e-mail: ayriyan@jinr.ru

$\star \star$ e-mail: yegorov@kapella.gpi.ru
} 
An investigation whether the origin of the observed effect originates in the electric field magnitude or in the pulse-periodic nature of the field is important. Moreover, it is interesting to study the spacemodulated LC structure formed by the field. In this paper, a two-dimensional mathematical model for the experimentally observed domains is proposed. The mathematical simulation of the director orientation in static electric fields in the side-electrode cell has been carried out. The Fredericks transition threshold for the central part of the cell, as well as dependencies of the distribution of the director orientation patterns on the electric field magnitude and location were obtained.

\section{Mathematical formulation of the problem}

The design of the LC cell is presented in fig. 1. The cell walls are glass platelets. The surfaces of the platelets are covered with surfactant providing LC homeotropic orientation, when long 5CB molecular axes are parallel to the $Y$-axis. A static electric field is directed along to the $X$-axis. It is supposed that only longitudinal and transverse bends of the director appear in the field and the change in the director orientation (director distortion) is defined by the angle $\theta$ between the director and the $Y$-axis at the equilibrium of the electric field and elasticity torques. Therefore, the angle $\theta$ in our case is a function of the $x$ and $y$ coordinates.

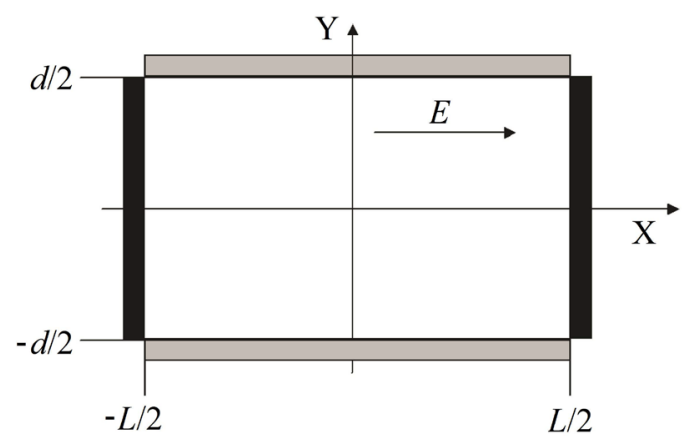

Figure 1. Cross-section of the cell used in the calculations. The electrodes are in black, glass platelets are in grey. The thickness of LC layer is $d$ and the inter-electrode distance is $L$.

The standard procedure of free energy density minimization togather with qualitative approximations for the simplification of the solution result in the nonliner differential equation,

$$
4 K\left(\theta_{x x}+\theta_{y y}\right)+0.5 \varepsilon_{0} \Delta \varepsilon E^{2} \sin (2 \theta)=0 .
$$

Here $K=0.5\left(K_{11}+K_{33}\right)$, with a splay elastic constant $K_{11}=7 \cdot 10^{-12} \mathrm{~N}$, a bend elastic constant $K_{33}=10^{-13} \mathrm{~N}$ [4], the dielectric constant of the vacuum $\varepsilon_{0}=8.854 \cdot 10^{-12} \mathrm{~F} / \mathrm{m}$, the dielectric anisotropy $\Delta \varepsilon$ [4], $\theta_{\alpha \alpha} \equiv \partial^{2} \theta / \partial \alpha^{2}$ with $\alpha=x, y$.

The definition domain is $\Omega=\{(x, y) \mid-L / 2 \leq x \leq L / 2,-d / 2 \leq y \leq d / 2\}$, with $L=2 \cdot 10^{-3} \mathrm{~m}$ and $d=10^{-4} \mathrm{~m}$. The boundary conditions are

$$
\theta(x,-d / 2)=0, \quad \theta(x, d / 2)=0, \quad \theta(-L / 2, y)=\pi / 2, \quad \theta(L / 2, y)=\pi / 2 .
$$

For convenience, the eq. (1) was converted to:

$$
\theta_{x x}+\theta_{y y}+q E^{2} \sin (2 \theta)=0,
$$

where $q=1.822882353 \mathrm{~V}^{-2}$.

Well-known finite-difference methods were used for the solution of the eq. (2) [5]. The programs for numerical solution of the two-dimensional parabolic partial differential equation were developed both in FORTRAN and C. 


\section{Results of Calculation and Discussion}

Calculated dependencies of LC director orientation on the electric field magnitude and location are shown in fig. 2. Fig. 2a unveils the existence of a threshold value of $1.6 \cdot 10^{3} \mathrm{~V} / \mathrm{m}$ of the electric field above which a sharp director rotation occurs. This value can be taken for the Fredericks effect threshold for two-dimensional deformation of LC director in static electric field. A director rotation angle $\theta=45^{\circ}$ is reached at the field value $E \sim 2 \cdot 10^{4} \mathrm{~V} / \mathrm{m}$. At $E \geq 5 \cdot 10^{4} \mathrm{~V} / \mathrm{m}$ the director is predominantly oriented along the field.

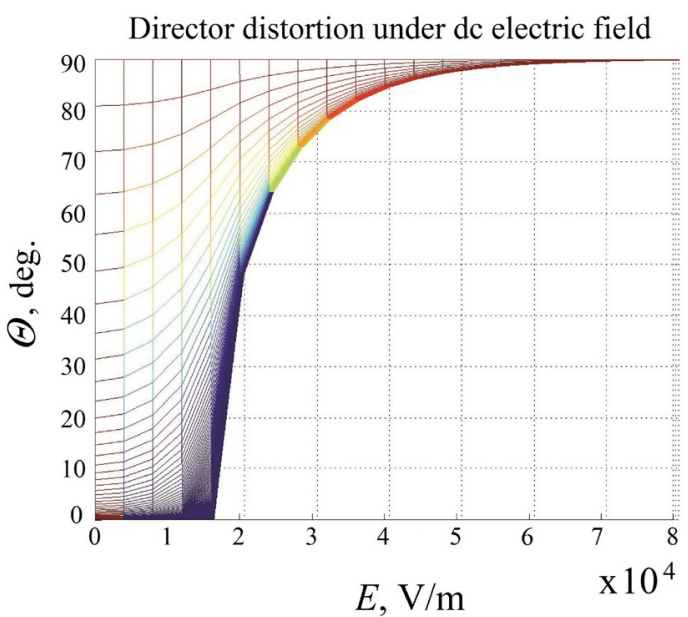

(a)

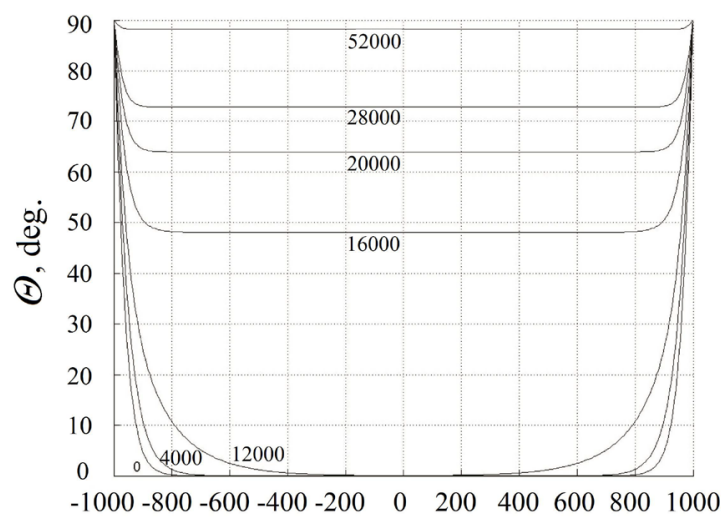

$L, \mathrm{mkm}$

(b)

Figure 2. (a) Dependence of NLC director orientation (angle $\theta$ ) on the static electric field at different positions between the electrodes (oblique lines). (b) Dependence of the director orientation (angle $\theta$ ) on the position between the electrodes at different electric field values $(\mathrm{V} / \mathrm{m})$.

Fig. $2 \mathrm{~b}$ points to the fact that a gradual change of the director orientation occures in regions close to the electrodes. The summed widths of these regions do not exceed $10 \%$ of the inter-electrode distance, they are of the order of the LC layer thickness $(d=100 \mathrm{mkm})$ irrespective of the field values. At the same time, in the central region the magnitude of the angle $\theta$ is almost constant at each given $E$, it increases with the field magnitude and reaches $90^{\circ}$ at the electrodes.

The size of the above-mentioned regions is important for the comparison with the experiment. Experimental images of LC cell under the influence of the pulse-periodic electric field are shown in fig. 3 [3]. Side dark domains are the electrodes (the inter-electrode distance of $2 \cdot 10^{-3} \mathrm{~m}$ corresponds to the respective parameter of the model), central dark domain with the varying width is the region of LC undistorted (initial) homeotropic orientation and two light domains with varying width are the regions of changed (distorted) director orientation. The left image (fig. 3a) was taken in the absence of the external field and the right one (fig. 3b) after the field was applied for $16 \mathrm{~min}$.

The width of the light stripes in fig. 3 a does not exceed $10 \%$ of the total inter-electrode distance. This is in good agreement with the calculation results at $E=0$. However in the fig. $3 \mathrm{~b}$ this width increases and reaches magnitudes from $15 \%$ to $30 \%$ of the distance. One can use some value of the angle $\theta$ as a boundary point, dividing "dark" and "light" regions according to their values of the angle $\theta$ (e.g., at $\theta<10^{\circ}$ - region is dark, at $\theta>10^{\circ}$ - region is light) to separate all points in the image into dark and light domains. Then in the interval $(0.4-1.6) \cdot 10^{4} \mathrm{~V} / \mathrm{m}$ the obtained angle patterns, formally, will have a qualitative agreement with the experiment. However a number of questions associated with the contrast of dark and light domains in fig. 3 remains open. 
One can see that the transition from one domain to another is sharp and all parts of light domain correspond to similar director orientation. At the same time it can be concluded from fig. $2 \mathrm{~b}$ that in the above-mentioned interval of the field the solution demonstrates:

1) smooth change of the angle around any value of $\theta$, and

2) strong changes of the angle $\theta$ (from $10^{\circ}$ to $90^{\circ}$ ) take place in near-electrode regions.

This indicates that the used two-dimensional model of LC cell and static electric field cannot completely account for the observed experimental effect, which is likely caused by the pulse-periodic nature of the applied experimental field. Further studies for the elucidation of this point are needed.

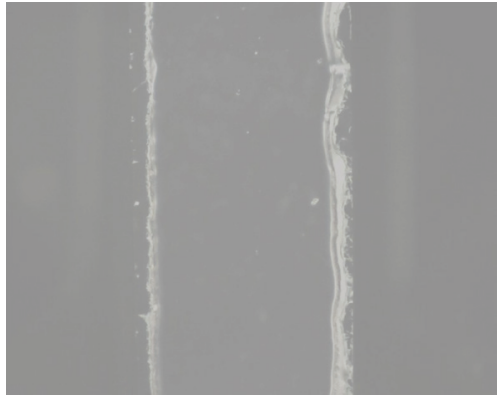

(a) field off

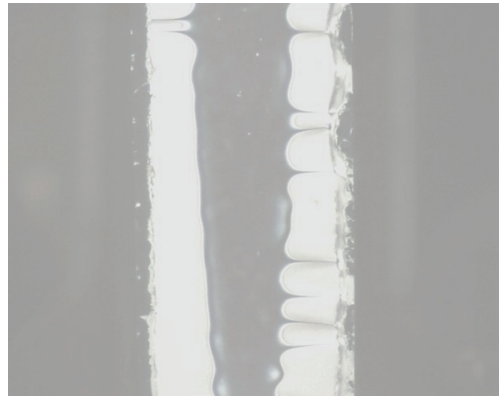

(b) field on

Figure 3. Changes of the director orientation in a cell with initially homeotropic oriented NLC, subjected to a pulse-periodic electric field [4].

\section{Summary}

A two-dimensional model of the Fredericks effect in side-electrode cell was used for the investigation of the NLC director distribution. Fredericks transition threshold for the central part of the cell as well as dependencies of the distribution of the director orientation on the location and electric field magnitude were obtained. The results of the calculation were compared with the experiment.

The calculations have shown that the used model of LC cell with side electrodes results in correct homeotropic NLC director orientation in the absence of an electric field. At the same time, the introduction of static electric field to the model cannot completely explain the observed experimental effect, which is, likely, due to the pulse-periodic nature of the applied experimental field.

Acknowledgements. E. A. Ayryan appreciates financial support of the Ministry of Education and Science of the Russian Federation (the Agreement number 02.a03.21.0008). A. S. Ayriyan thanks the JINR grant (project no. 17-602-01).

\section{References}

[1] I. Ch. Khoo, Liquid Crystals (Wiley Interscience, New York, 2007) 384 pp.

[2] Y. G. Marinov, G. B. Hadjichristov, A. G. Petrov, S. Sridevi, U. S. Hiremath, C. V. Yelamaggad, and S. K. Prasad, Journal of Physics CS 253, 012060 (2010)

[3] A. S. Ayriyan, E. A. Ayrjan, A. A. Egorov, G. B. Hadjichristov, Y. G. Marinov, I. A. Maslyanitsyn, A. G. Petrov, J. Pribis, L. Popova, V. D. Shigorin, A. Strigazzi, and S. I. Torgova, Physics of Wave Phenomena 24 (4), 259 (2016)

[4] A. Bogi and S. Faetti, Liq. Cryst. 28 (5), 729 (2001)

[5] A. A. Samarsky, The Theory of Difference Schemes (Marcel Dekker Inc., New York, 2001) 788 pp. 